\title{
Elevated plasma chitotriosidase activity in various lysosomal storage disorders
}

\author{
Yufeng Guo ${ }^{1}$, WAng He ${ }^{1}$, A. M. Boer ${ }^{1}$, R. A. Wevers ${ }^{2}$, A. M. De Bruijn ${ }^{3}$, \\ J. E. M. GROENER ${ }^{4}$, C. E. M. HollaK ${ }^{5}$, J. M. F. G. AERTS ${ }^{5}$, H. GALJAARD ${ }^{1}$ and \\ O. P. VAN DigGELEN ${ }^{1 *}$ \\ 'Department of Clinical Genetics, Erasmus University, Rotterdam; ${ }^{2}$ Institute of \\ Neurology, University Hospital Nijmegen; ${ }^{3}$ Department of Epidemiology \& Biostatistics, \\ Erasmus University, Rotterdam; ${ }^{4}$ Clinical Genetics Centre, University of Leiden; \\ ${ }^{5}$ Department of Biochemistry, Academic Medical Centre, Amsterdam, The Netherlands \\ *Correspondence: Department of Clinical Genetics, Erasmus University, P.O. Box 1738, \\ 3000 DR Rotterdam, The Netherlands
}

MS received 21.3.95 Accepted 8.8.95

\begin{abstract}
Summary: Recently a striking elevation of the activity of chitotriosidase, an endo $\beta$-glucosaminidase distinct from lysozyme, was found in plasma from patients with Gaucher type I disease (McKusick 230800). Plasma chitotriosidase originates from activated macrophages and this elevation is secondary to the basic defect in Gaucher disease. To investigate the specificity of this phenomenon, we have investigated 24 different lysosomal storage diseases. In 11 different diseases increased chitotriosidase activity in plasma was found (in $28 \%$ of the patients). None of these diseases showed elevations as high as in Gaucher disease. Chitotriosidase was not significantly elevated in plasma from 20 different non-lysosomal enzymopathies or in plasma from patients with infectious diseases associated with hepatomegaly. The results show that marked elevation of chitotriosidase activity in plasma appears to be specific for Gaucher disease. The data further suggest that elevated levels of chitotriosidase activity in plasma from patients with unexplained diseases may be indicative for a lysosomal disorder.
\end{abstract}

Recently it was demonstrated that plasma of patients with Gaucher disease had 100-1000fold elevated activity of chitotriosidase (Hollak et al 1994). Chitotriosidase is probably identical to a previously described chitotetraosidase, an endo $\beta$-glucosaminidase distinct from lysozyme (Den Tandt et al 1988) which is secreted by activated macrophages (Hollak et al 1994). The elevation in Gaucher disease is secondary to the well established primary defect in this disease: a deficiency of the lysosomal hydrolase $\beta$-glucosidase (for review see Beutler and Grabowski 1995).

We were interested whether this novel marker for Gaucher disease would also be useful in detecting other (metabolic) disorders. Here we report elevated levels in 11 different lysosomal storage disorders. 


\section{MATERIALS AND METHODS}

Chitotriosidase activity was determined by incubating $5 \mu \mathrm{l}$ plasma or serum with $100 \mu \mathrm{l}$ $22 \mu \mathrm{mol} / \mathrm{L}$ 4-methylumbelliferyl- $\beta$-D- $N, N^{\prime}, \mathrm{N}^{\prime \prime}$-triacetylchitotriose (MU-( $\beta$-GlcNAc) $)_{3}$; Sigma) in Mcllvain's phosphate-citrate buffer, $\mathrm{pH} 5.2$, for $1 \mathrm{~h}$ at $37^{\circ} \mathrm{C}$ (modified from Hollak et al 1994). Reactions were terminated by adding $120 \mu \mathrm{l} 0.5 \mathrm{~mol} / \mathrm{L} \mathrm{Na}_{2} \mathrm{CO}_{3}-$

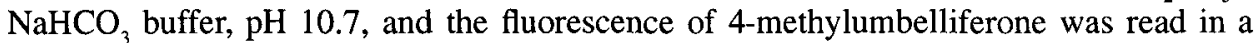
Fluoroskan fluorimeter (Titertek; excitation $355 \mathrm{~nm}$, emission $460 \mathrm{~nm}$ ).

Chitotriosidase was also determined in leukocytes and cultured fibroblasts by substituting plasma for sonicated homogenates containing $20 \mu \mathrm{g}$ protein.

\section{RESULTS}

Kinetic properties and origin of plasma chitotriosidase: The apparent $K_{\mathrm{m}}$ for MUchitotrioside could not be determined since chitotriosidase showed strong substrate inhibition at concentrations above $0.1 \mathrm{mmol} / \mathrm{L}$. Maximal activity was obtained at a MU$(\beta \text {-GlcNAc) })_{3}$ concentration of $20 \mu \mathrm{mol} / \mathrm{L}$. At this substrate concentration the activity increased linearly with incubation times up to $90 \mathrm{~min}$ and the $\mathrm{pH}$ optimum was 5.2 (data not illustrated).

Stability of chitotriosidase: EDTA and heparin plasma, stored for up to 8 years at $-80^{\circ} \mathrm{C}$ in three different laboratories, as well as serum samples stored for 20 years at $-20^{\circ} \mathrm{C}$ were used. The effect of anticoagulant, storage, and freeze-thawing on chitotriosidase activity was tested. Heparin and EDTA plasma samples from 82 blood donors did not show significantly different activities. Likewise, heparin plasma and serum from 20 individuals showed the same activity. Ten cycles of freezing at $-80^{\circ} \mathrm{C}$ and thawing had no effect on the chitotriosidase activity in plasma or serum. Recently obtained plasma samples from blood donors ( 84 male, 84 female) aged 21-61 years were divided into four age groups of 10 years (21 persons/group) and matched to serum samples that had been stored for 20 years at $-20^{\circ} \mathrm{C}(21-30$ persons/group). Chitotriosidase in the matched groups did not differ significantly. The mean activity in 168 plasma samples did not differ significantly from the mean of 32 plasma samples from blood donors that had been stored for 7 years at $-80^{\circ} \mathrm{C}$ ( 4 persons/group). These results indicate that chitotriosidase is a very stable enzyme, allowing direct comparison of plasma and serum samples that have been stored under widely different conditions. Freeze drying of plasma had no effect on chitotriosidase activity and storage of the dried plasma for 3 weeks at room temperature led to $15 \%$ loss of enzyme activity.

Effect of age and sex on chitotriosidase activity: Age-matched samples from 86 male and 88 female blood donors (aged 21-65 years) were compared. Chitotriosidase activity did not differ significantly. Linear regression analysis of the data, however, showed a clear effect of age on chitotriosidase activity $(p<0.003)$ : the activity increased with age. Since most of our patients are under 20 years of age, we could not use the blood donors as reference group. 


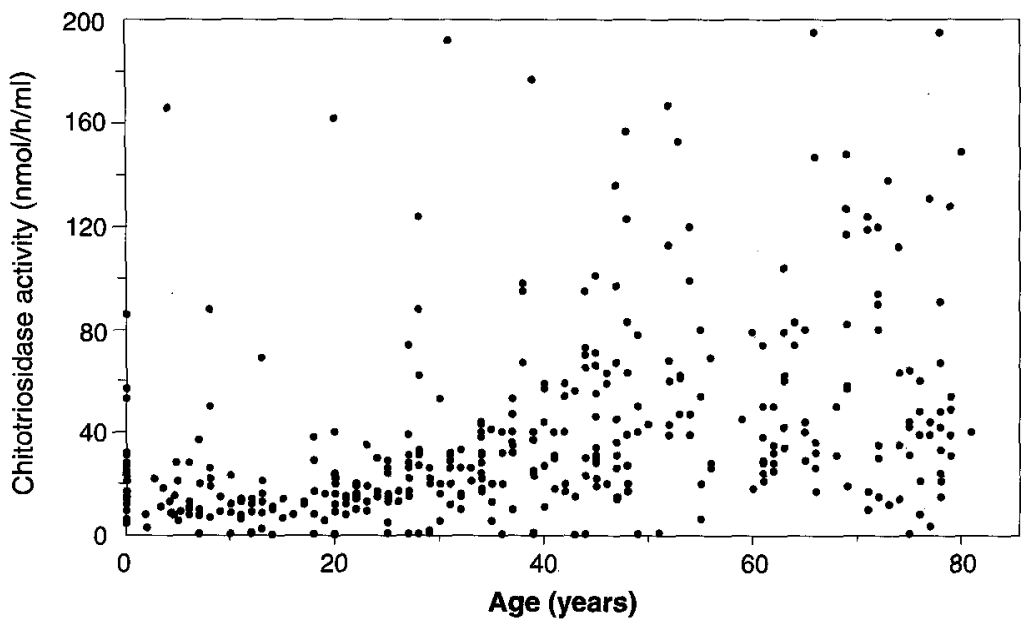

Figure 1 Chitotriosidase activity in serum or plasma from 385 control individuals in the age range $0-81$ years

Table 1 Plasma chitotriosidase activities and deficiencies in controls and Gaucher patients

\begin{tabular}{lcc}
\hline & Control activity $^{\mathrm{a}}$ & Deficiency \\
\hline Blood donors, male age 21-65 years $(n=86)$ & $4-157$ & $0.2-2.2(n=5)$ \\
Blood donors, female age 22-64 years $(n=88)$ & $6-142$ & $0.1-1.8(n=4)$ \\
Controls age 0-81 years $(n=385)$ & $4-195$ & $0.3-1.9(n=24)$ \\
Gaucher type I $(n=504)^{\mathrm{b}}$ & $1980-69900$ & $0-1.7(n=30)$ \\
\hline
\end{tabular}

${ }^{\text {aRange; }}$ activities expressed as nmol/h per ml plasma

'Samples collected during a worldwide collaborative study of laboratory 5 , hence the high number

Reference values of chitotriosidase activity in the general population: Chitotriosidase was determined in 26 plasma samples from cord blood and in 359 samples out of about 5000 sera, which were collected during an epidemiological survey in a Dutch town. The ages ranged between 3 and 81 years (163 women and 186 men; Figure 1 and Table 1). After logarithmic transformation, the chitotriosidase values were approximately normally distributed. Linear regression analysis showed an age-dependent increase of chitotriosidase activity $(p<0.001)$.

The variation of activity was analysed statistically and chitotriosidase activities for which the absolute difference from the mean, after correction for age, was greater than twice the standard deviation were regarded as outliers. Using these criteria, 24 persons $(6.2 \%)$ had very low chitotriosidase activities between 0.3 and 1.9 (outliers with chitotriosidase deficiency). Among Gaucher patients a similar percentage of deficient individuals was found (5.9\%; Table 1). The presence of a diffusible inhibitor of chitotriosidase in deficient individuals was ruled out by mixing normal and deficient samples. These observations indicated heterogeneity in the general population and that about $6 \%$ has a chitotriosidase deficiency. Only 3 samples $(0.8 \%$; Figure 1$)$ had chitotriosidase activities which were 'too high' (outliers with very high activity); the reference enzyme $\alpha$-mannosidase was not 
Table 2 Plasma chitotriosidase activity in 24 lysosomal disorders ${ }^{a}$

\begin{tabular}{lcc}
\hline & \multicolumn{2}{c}{ Plasma chitotriosidase activity } \\
\cline { 2 - 3 } Disease & Abnormalltotal & $\begin{array}{c}\text { Elevated activity } \\
\text { (nmol/h per ml) }\end{array}$ \\
\hline Aspartyl glucosaminuria & $0 / 3$ & \\
Fabry & $0 / 8$ & $5580-51800$ \\
Gaucher type I, & $20 / 21$ & $360 ; 420$ \\
Glycogen storage disease II & $2 / 8$ & $380 ; 720-1420$ \\
GM1-gangliosidosis ${ }^{\mathrm{b}}$ & $7 / 13$ & $610-1670$ \\
GM2-gangliosidosis & $0 / 11$ & 300 \\
Krabbe disease ${ }^{\mathrm{b}}$ & $7 / 11$ & 550 \\
$\alpha$-Mannosidosis & $1 / 3$ & $600 ; 400$ \\
$\beta$-Mannosidosis & $0 / 2$ & \\
Metachromatic leukodystrophy & $1 / 29$ & \\
Mucopolysaccharidoses & $2 / 63$ & \\
(I; II; IIIA; B, C; IVA, B) & & \\
Mucolipidosis II/III & $0 / 3$ & $250 ; 602-2800$ \\
$\alpha-N A G A$ deficiency & $0 / 2$ & $263 ; 304-940$ \\
Niemann-Pick A/B & $13 / 15$ & \\
Niemann-Pick C & $6 / 11$ & $(28 \%)$ \\
Sialic acid storage disorders & $0 / 1$ & \\
Sialidosis & $0 / 1$ & \\
Total & $58 / 205$ & \\
\hline
\end{tabular}

'The patients in this table were diagnosed in the authors' laboratories (Rotterdam, Nijmegen and Leiden) and reflect the relative frequencies of the various diseases. The number of Gaucher patients is therefore different from the high number in Table 1

'Diseases where majority of patients have elevated chitotriosidase activity

elevated in these samples. In the following studies chitotriosidase activity was regarded as elevated if it was higher than the highest reference value (i.e. $>195 \mathrm{nmol} / \mathrm{h}$ per $\mathrm{ml}$ ).

Chitotriosidase activity in leukocytes and fibroblasts: Leukocytes and cultured fibroblasts were available from two individuals with a chitotriosidase deficiency in plasma. The activity in leukocytes was also deficient $(0.0$ and $0.2 \mathrm{nmol} / \mathrm{h}$ per $\mathrm{mg}$; control range $110-$ $280, n=8$ ) whereas the fibroblasts had normal chitotriosidase activity ( 3.4 and $4.1 \mathrm{nmol} / \mathrm{h}$ per $\mathrm{mg}$; control range $3.2-14, n=11$ ). This indicates identity of the plasma and leukocyte chitotriosidase and non-identity of the plasma and fibroblast chitotriosidase.

Chitotriosidase activity in inherited metabolic disorders: Chitotriosidase activity in plasma samples from 23 different lysosomal storage disorders was determined and considered elevated if it was greater than the highest reference value $(195 \mathrm{nmol} / \mathrm{h} \mathrm{per} \mathrm{ml}$; Figure 1). In 10 diseases other than Gaucher disease, slight to moderate elevations of chitotriosidase activity were observed (Table 2). In 4 of these diseases, all sphingolipidoses, the majority of the patients had elevated activity. In one Niemann-Pick type A patient, chitotriosidase activity was followed over a period of 3 months. At the time of diagnosis ( 3 months of age) chitotriosidase was not elevated $(118 \mathrm{nmol} / \mathrm{h}$ per $\mathrm{ml}) ; 1.5$ 
Table 3 Plasma chitotriosidase activity in other metabolic and infectious disorders

\begin{tabular}{lcc}
\hline & \multicolumn{2}{c}{ Plasma chitotriosidase activity } \\
\cline { 2 - 3 } Disease & Abnormal/total & $\begin{array}{c}\text { Elevated activity } \\
\text { (nmol/h per ml) }\end{array}$ \\
\hline Adrenoleukodystrophy & $0 / 1$ \\
Canavan disease & $0 / 1$ \\
Carbohydrate-deficient glycoprotein syndrome & $0 / 3$ \\
Chondrodysplasia punctata & $0 / 2$ \\
LCAD deficiency & $0 / 1$ \\
Fructose-1,6-bisphosphatase deficiency & $0 / 2$ \\
Glutaric acidaemia I & $0 / 3$ \\
Galactosaemia & $0 / 4$ \\
Glycogen storage disease III & $0 / 3$ \\
Glycogen storage disease IV & $0 / 1$ \\
Glycogen storage disease V & $0 / 2$ \\
Glycogen storage disease VI & $0 / 2$ \\
Glycogen storage disease VIII & $0 / 5$ \\
G6PD deficiency & $0 / 1$ \\
Ichthyosis, X-linked & $1 / 6$ \\
Lesch-Nyhan syndrome & $0 / 3$ \\
Molybdenum cofactor deficiency & $0 / 1$ \\
Propionic acidaemia & $0 / 4$ \\
Pyruvate carboxylase deficiency & $0 / 1$ \\
Zellweger syndrome & $0 / 1$ \\
Infectious diseases with hepatomegaly & $0 / 12$ \\
& \\
Total & $1 / 45$ \\
\hline
\end{tabular}

months later it had risen to 278 units and at 6 months of age it was strongly elevated (772 units).

As a reference, samples from patients with 21 different non-lysosomal diseases were examined; only one patient had marginally elevated activity (Table 3 ). Infectious diseases with hepatomegaly did not lead to elevated chitotriosidase activity (Table 3 ).

Chitotriosidase activity in undiagnosed patients suspected of a lysosomal disorder: Plasma from patients referred for enzyme analysis for lysosomal disorders but remaining undiagnosed was also examined. In 16 out of 486 patients, chitotriosidase was elevated (range 350-1570 units, patients aged $1-70$ years).

\section{DISCUSSION}

The physiological role of chitotriosidase is not yet known. Since it is secreted by activated macrophages (Hollak et al 1994), a role as chitinase in the defence against chitincontaining pathogens seems likely. It is remarkable that about $6 \%$ of the population, healthy persons and patients alike, have a chitotriosidase deficiency. This is most clearly illustrated by Gaucher type I patients, who have greater than 100-fold elevated activities of chito- 
triosidase, except about $6 \%$ who also have a complete chitotriosidase deficiency. Limited family data suggest that this is an autosomal recessive trait. The percentage of deficiencies in our reference group (559 persons) appeared to decrease with increasing age. The Mann - Whitney test indicated that this difference was not significant. Whether chitotriosidase deficiency predisposes for certain infectious diseases is presently under investigation.

Chitotriosidase activity was considered elevated if it was greater than the highest value $(195 \mathrm{nmol} / \mathrm{h}$ per $\mathrm{ml})$ of 559 reference samples. We have included the three values which statistically were considered to be outliers, in order to avoid overlap between patients and controls. Among 21 different lysosomal disorders, only Gaucher type I disease is associated with very marked elevations of chitotriosidase activity in plasma (100-5000-fold). This elevation does not correlate with the well-known elevation of acid phosphatase activity in Gaucher disease (Hollak et al 1994). In 10 other lysosomal disorders, small to moderate increases of chitotriosidase activity were found. In 4 of these diseases more than two-thirds of the patients showed elevations. These 4 diseases and Gaucher disease are sphingolipidoses, which suggests that certain, but not all, glycosphingolipids may be able to stimulate macrophages to produce chitotriosidase. Limited data (one Niemann-Pick type A patient) suggest that chitotriosidase gradually increases with age, or with severity of the disease.

Chitotriosidase activity was not elevated significantly in plasma from 20 different nonlysosomal enzymopathies or in plasma from patients with infectious diseases with hepatomegaly. On the other hand, elevated activities were found in as many as $3 \%(16 / 486)$ of undiagnosed patients screened for a lysosomal disorder. It is unlikely that all these patients would still have undetected lysosomal disorders. However, our results suggest that these patients should be re-evaluated with special attention for lysosomal disorders. We have been unable to correlate elevated chitotriosidase activity with specific clinical signs and symptoms of these undiagnosed patients. The four highest had encephalopathy with perivasculitis of the retina combined with multifocal hypodensities of the white matter, limb-girdle dystrophy, organic psychotic syndrome or mental retardation without organomegaly and brain abnormalities on MRI.

\section{ACKNOWLEDGEMENTS}

We gratefully acknowledge Dr L.A. Sandkuijl for statistical analysis of the data and the bloodbanks of Rotterdam (Dr D.J. van Rhenen) and Nijmegen (Dr H. Olthuis) for providing indispensable blood samples from donors. Drs H.J. Aanstoot and M.R. Batstra are acknowledged for kindly making available serum samples from a screening programme among healthy individuals. We thank Drs J.W. Weber and W.C.G. Plandsoen from the AMC in Amsterdam for providing some of the samples from patients with Niemann-Pick C disease, and Ms I. van den Berg for swift technical assistance.

\section{REFERENCES}

Beutler E, Grabowski GA (1995) Gaucher disease. In Scriver CR, Beaudet WS, Sly WS, Valle D, eds. The Metabolic and Molecular Bases of Inherited Disease, 7th edn. New York: McGraw-Hill. $2641-2670$.

Den Tandt WR, Inaba T, Verhamme I, Overdijk B, Brouwer J, Prieur D (1988) Non-identity of human plasma lysozyme and 4-methylumbelliferyl-tetra- $N$-acetyl- $\beta$-D-chitotetraoside hydrolase. Int Biochem. 20: 713-719.

Hollak CEM, van Weely S, van Oers MHJ, Aerts JMFG (1994) Marked elevation of plasma chitotriosidase activity. A novel hallmark of Gaucher disease. J Clin Invest 93: 1288 - 1292. 